\title{
Nanoparticules ef couleur, une tradition millénaire
}

\author{
Philippe COLOMBAN \\ MONARIS - UMR 8233 CNRS \\ Université Pierre et Marie Curie Paris 6, Sorbonne Universités, Paris \\ philippe.colomban@upmc.fr
}

La puissance de coloration des nanoparticules de cuivre, une découverte des Celtes, fut largement utilisée dans les tesselles de mosaïque et objets en verre Romains. Les premiers dispositifs photoniques, les lustres céramiques bleu, vert, rouge, argenté ou doré, furent réalisés en Mésopotamie par les potiers Abbassides. En Occident le Moyen Âge vit le développement des verres jaunes à base de nanoparticules d'argent, puis à la Renaissance les nanoparticules à base d'or élargirent la palette au pourpre.

\section{Maîtriser la couleur}

Plusieurs mécanismes concourent à colorer un milieu. Un agent colorant, c'est-à-dire absorbant sélectivement une partie du spectre visible, conduit à observer la couleur complémentaire. L'agent colorant peut être présent en surface ou en volume. L'association de plusieurs agents permet un large choix de teintes. Un tout autre mécanisme de coloration est la diffraction : une hétérogénéité dont la période est proche de la longueur d'onde d'illumination donne naissance à des interférences aux couleurs intenses. La première méthode de coloration est la plus commune, les agents colorants étant soit des ions de transition (couches électroniques $3 d$ ), soit des ions de la séries des lanthanides (couches $4 f$ ), soit des colorants organiques (à base de cycles aromatiques, de liaison C-N ou à transfert de charge). La seconde méthode est moins usitée bien que de nombreux composés naturels soient colorés par diffraction : opales, ailes de papillons, carapaces de scarabées... La dispersion de nanoparticules métalliques dans une matrice peu absorbante permet de colorer la matière par ces deux méthodes.

\section{Rouge de cuivre, jaune $d^{d}$ 'argent et pourpre d'or}

L'utilisation de nanoparticules de cuivre pour colorer un silicate vitreux fut développée par les Celtes, principalement pour des émaux sur métal (Colomban, 2009).

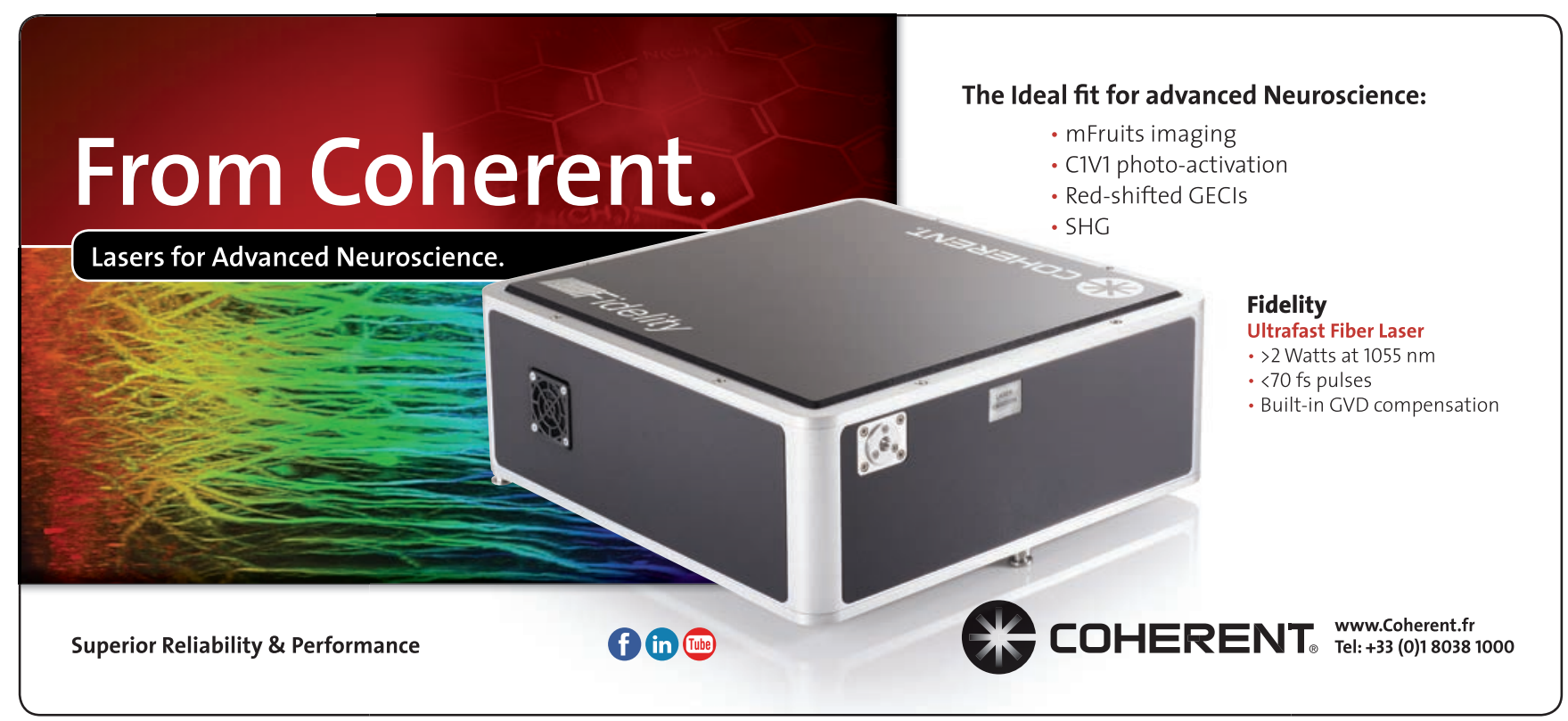



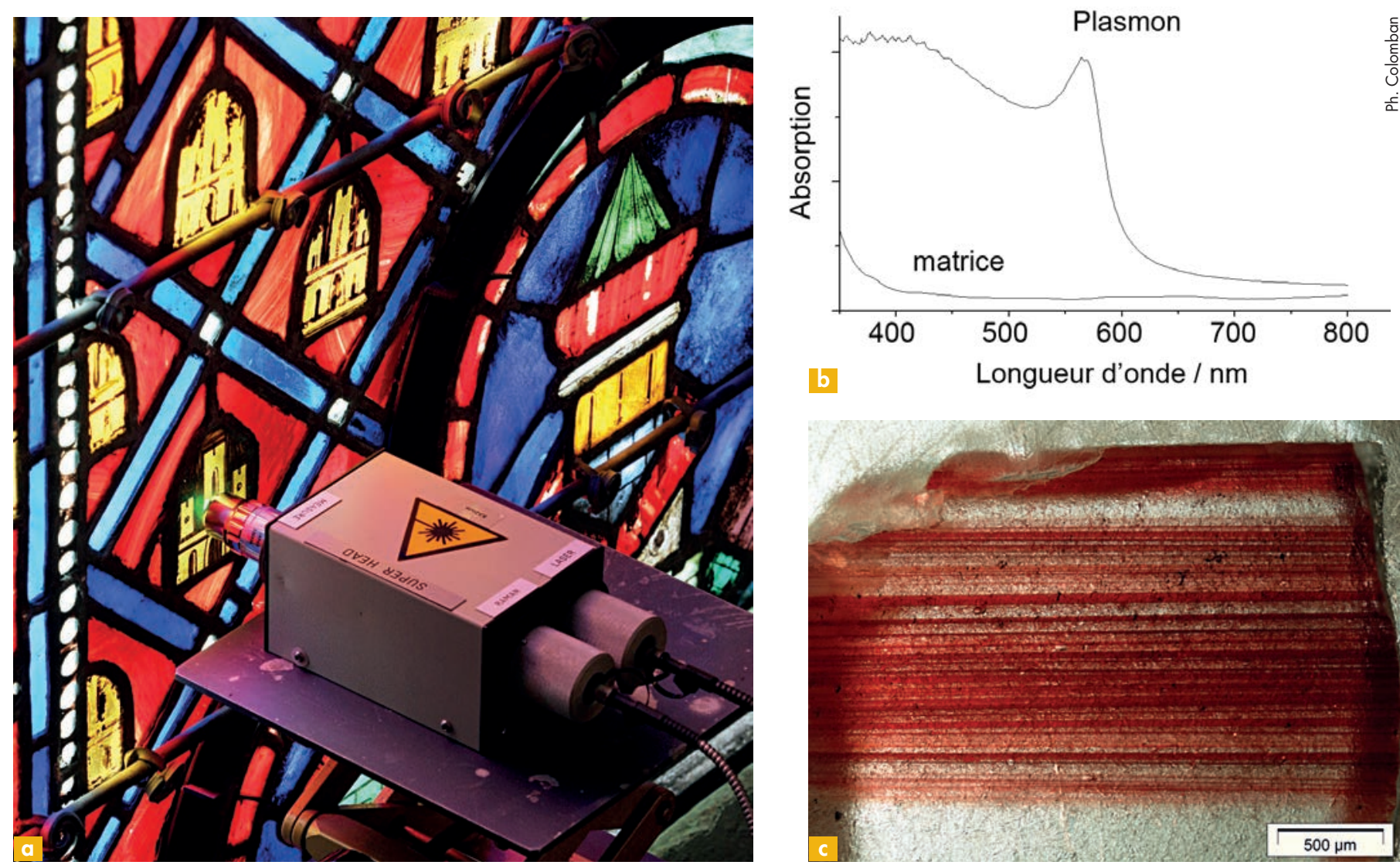

Figure 1. Les couleurs jaune et rouge du vitrail (Sainte-Chapelle, Paris, Xlle siècle) sont obtenues respectivement avec des nanoparticules d'argent et de cuivre (a). La très forte absorption des nanoparticules (b) conduit les verriers à alterner des dizaines de fines couches de verre, coloré ou non, sur un support transparent $(c$, le morceau de verre est posé sur un papier de soie).

Cette technique fut très largement utilisée par les Romains pour réaliser aussi bien des tesselles de mosaïque que des objets en verre. L'obtention de cuivre métallique nécessite un traitement thermique réducteur. En effet selon l'état d'oxydation du cuivre un verre contenant du cuivre sera turquoise $\left(\mathrm{Cu}^{2+}\right)$, incolore $\left(\mathrm{Cu}^{+}\right)$ou rouge $\left(\mathrm{Cu}^{\circ}\right)$. La réduction d'un ion dissous dans un verre fondu est difficile et est obtenue par 2 mécanismes, l'un direct, la diffusion d'hydrogène, l'autre indirect : l'atmosphère réductrice d'un four (riche en $\mathrm{CO}$ ) réduit tous les ions multivalents proches de la surface $\left(\mathrm{Fe}^{3+} / \mathrm{Fe}^{2+}, \mathrm{Sb}^{5+} / \mathrm{Sb}^{3+}\right.$, $\left.\mathrm{Sn}^{4+} / \mathrm{Sn}^{2+}, \mathrm{Bi}^{5+} / \mathrm{Bi}^{3+}, \mathrm{Eu}^{3+} / \mathrm{Eu}^{2+} . ..\right)$; ces ions diffusent facilement et réduisent à l'état métallique, dans le volume du verre fondu, par équilibre redox d'autres ions comme $\mathrm{Cu}^{2+} / \mathrm{Cu}^{+}, \mathrm{Ag}^{+}$ou $\mathrm{Au}^{+} / \mathrm{Au}^{3+}$.

La figure 1 a montre un vitrail de la SainteChapelle (sur I'lle de la Cité, à Paris) aux couleurs de Blanche de Castille, la mère du roi Saint-Louis. Cette chapelle Palatine fut édifiée pour conserver les reliques de la Passion du Christ, ramenées d'Orient. Le rouge est obtenu par l'absorption très forte en dessous de $600 \mathrm{~nm}$ du cuivre $\left(\mathrm{Cu}^{\circ}\right)$, avec le pic de l'absorption par le plasmon ( «gaz » d'électrons de la nanoparticule) se superposant à l'absorption des transitions électroniques inter-bandes (figure $1 b$ ). Typiquement 0,25\% en masse de cuivre métallique rend opaque un verre épais de $1 \mathrm{~mm}$ ! Aussi afin de garder une bonne luminosité, les verriers du Moyen Âge réalisaient du verre "flashé », en alternant l'éclatement de bulles de verre contenant ou non du cuivre pour former une succession de couches de quelques microns à dizaines de microns d'épaisseur (figure 1c).

L'absorption des nanoparticules d'argent, aussi très forte, est observée en dessous de $400 \mathrm{~nm}$ ce qui donne la couleur jaune (Colomban, 2009). Pour limiter le nombre de nanoparticules et leur absorption, une technique de cémentation est utilisée depuis le Moyen Âge
(Colomban, 2009) : des sels d'argent sont déposés sur une des surfaces du verre ; un traitement thermique provoque leur diffusion dans le verre où la combinaison de l'atmosphère réductrice et des équilibres redox conduit à la précipitation de nanoparticules d'argent. L'inconvénient de cette technique est la facile corrosion par les eaux de pluie de la face traitée si elle est positionnée coté extérieur, cette corrosion obscurcissant le vitrail.

L'utilisation documentée de nanoparticules d'or pour colorer le verre en pourpre ou rubis (absorption en dessous de $550 \mathrm{~nm}$ ) est plus tardive. Elle est attribuée selon les auteurs et le type de matrice, verre ou émail, à Johann Kunckel (1630-1703), Bernard Perrot (1619-1709), Johan Rudolf Glauber (1604-1670) ou Andreas Cassius (1605-1673). Des analyses locales en microscopie électronique de la Coupe de Lycurgus datée du IV ${ }^{\mathrm{e}}$ siècle et conservée au British Museum, fameuse pour son caractère dichroïque (verte en 

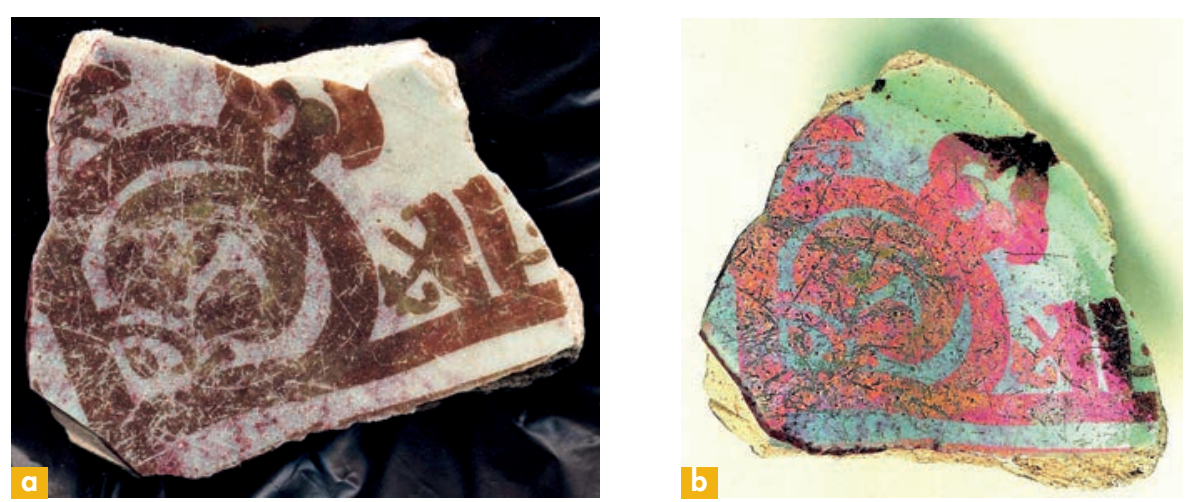

$<$

\section{diffraction}

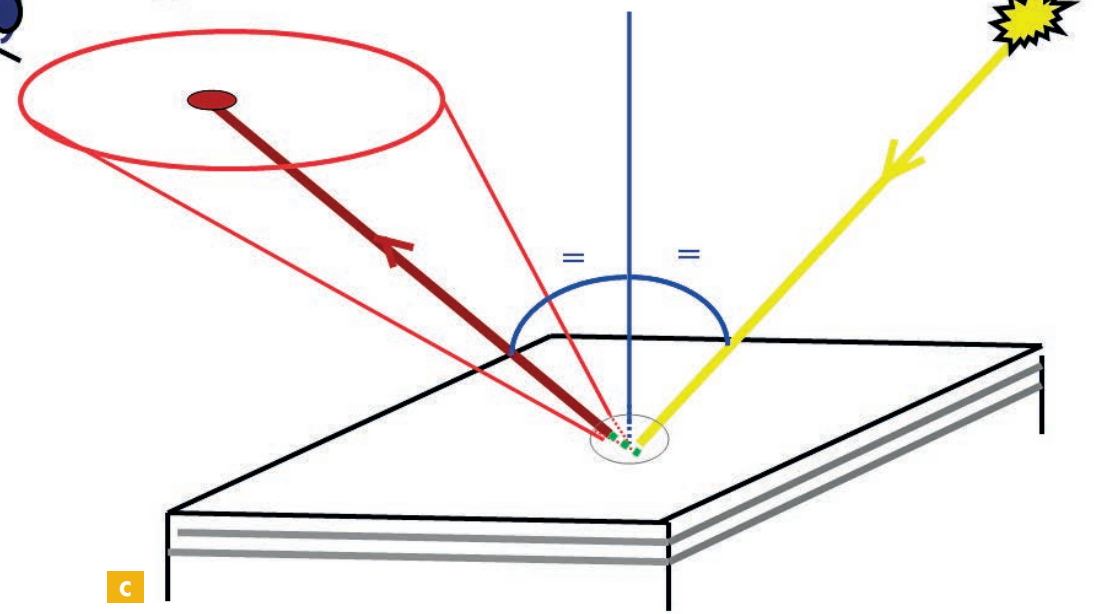

Figure 2. Le décor marron (a) en palmette de ce tesson lustré Fatimide (Fustat, Égypte, XIIle siècle) apparaît rouge ou doré (b) en fonction de l'angle de diffraction (c) ; certains tessons Abbassides (IXe siècle) (d) possèdent exceptionnellement un lustre où la polychromie est ajustée au décor.

réflexion, rouge en transmission), ont attribué sa coloration à la présence de particules d'or contenant un peu d'argent. Mais les analyses globales montrent dix fois plus de cuivre que d'or ! Il est donc raisonnable de penser que comme les autres verres Romains rouges (Colomban, 2009) la coupe est colorée majoritairement par des particules de cuivre, méthode classique pour obtenir des verres dichroïques (Colomban \& Schreiber, 2005).

Le pourpre de Cassius, obtenu à partir d'or colloïdal préparé par précipitation du chlorure d'or en présence d'étain, fut très utilisé au XVIII ${ }^{\mathrm{e}}$ siècle dans les émaux des premières porcelaines européennes. Son fort pouvoir colorant est apprécié pour la réalisation d'émaux de faible épaisseur, quelques dizaines de microns.

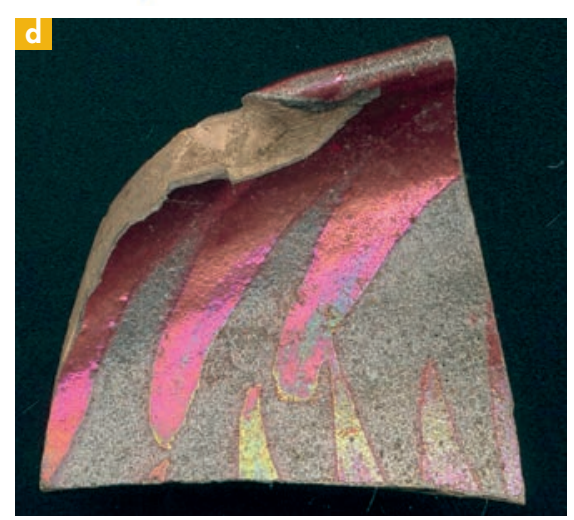

\section{Les céramiques lustrées, premiers dispositifs photoniques}

Un lustre céramique se caractérise par une coloration iridescente, c'est-à-dire un reflet pour un angle particulier d'observation (figure 2) : ainsi la palmette du tesson Fatimide apparaît marron pour l'essentiel des angles d'observation (figure 2a) mais pour un angle particulier un reflet rouge et doré est observé (figure 2b).

Les anciens recueils techniques rapportent l'existence de verres Romains aux

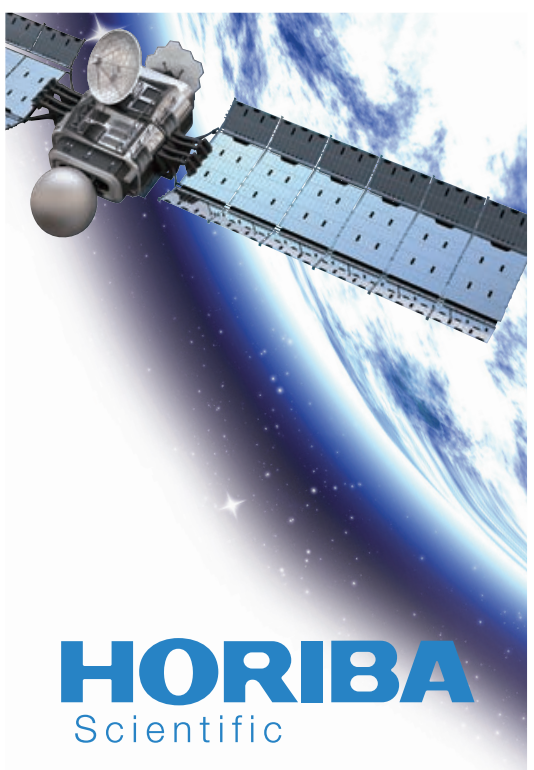

Des composants

et des

instruments

optiques

qui construisent

le futur

horiba.com/annee-de-la-lumiere

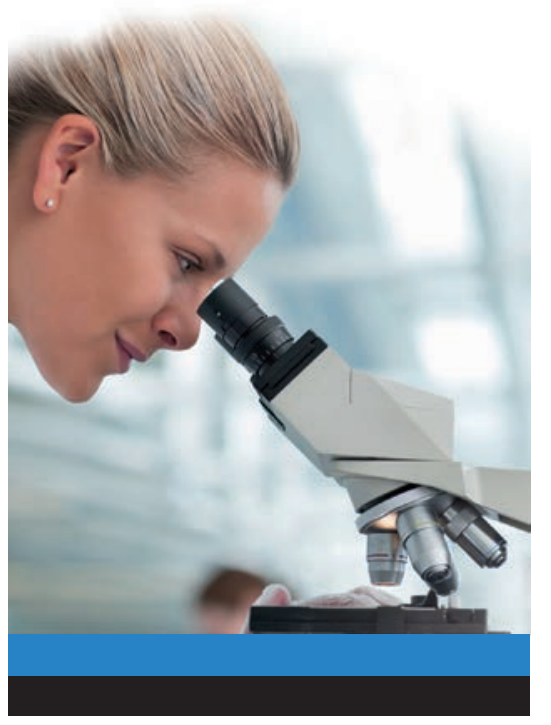




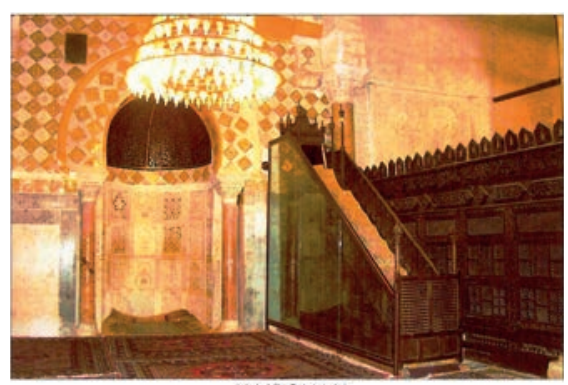

KAIROUAN

Figure 3. Vue ancienne (début XXe siècle) du Mihrab de la mosquée de Kairouan.

couleurs changeantes (Colomban, 2004 ; 2009) mais il semble qu'aucun objet de ce type n'a été trouvé et encore moins analysé. Des verres qualifiés de lustrés sont réputés avoir été produits par des artisans

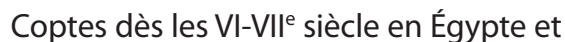
en Syrie. Nos rares analyses de tels objets, beaucoup plus tardifs, montrent une technique de type cémentation sans réalisation d'un lustre à proprement parler. Les véritables céramiques lustrées apparaissent être une invention des potiers Abbassides, à Baghdâd, Sâmarrâ ou Suze, en Mésopotamie : la figure 3 montre une vue ancienne des exceptionnels carreaux lustrés polychromes du Mihrâb de la mosquée aghlabide Sidi Oqba de Kairouan (Tunisie), importés en 862 de Baghdâd d'après les textes. La figure $2 d$ montre un tesson polychrome (voir d'autres photos: Colomban, 2004).
La technique s'est ensuite disséminée vers l'Égypte, durant la dynastie Tûlûnide (868905) qui se voulait rivale de Baghdâd et Sâmarrâ. Au début de la période Fâtimide (909-1171), les objets présentent un très beau décor essentiellement animalier et figuratif. L'Iran (Kashan) et l'Anatolie produisent ensuite des très beaux lustres monochromes et la technique diffuse dans tout le monde islamique pour atteindre I'Espagne (al Andalous) à l'époque des califes Umayyade (929-1031). Sous la dynastie Almoravide (1056-1147) mais plus vraisemblablement Almohade (11471269), la production de décors lustrés à Murcie, Malaga, Siyasa, Paterna-Manises (Valence) se développe et reste importante jusqu'au XVIIe siècle. À la fin du $X V^{e}$ siècle, la technique se répandit, sous le nom de Majolique, en Italie centrale dans des sites comme Deruta, Gubbio, Caffaggiolo, pour produire une vaisselle au décor reprenant celui de la peinture et à reflet métallique. À la fin du XVIII' siècle à la suite de J. Wedgwood en Angleterre mais surtout dans la deuxième moitié du XIX siècle l'intérêt des potiers chimistes (Théodore Deck par exemple) vers les technologies anciennes fut à l'origine d'un renouveau d'intérêt pour le lustre : créations de Vilmus Zsolnay et Köhler en Europe Centrale, de William de Morgan en Angleterre et de Clément Massier ou Jean Mayodon en France. L'intérêt reste

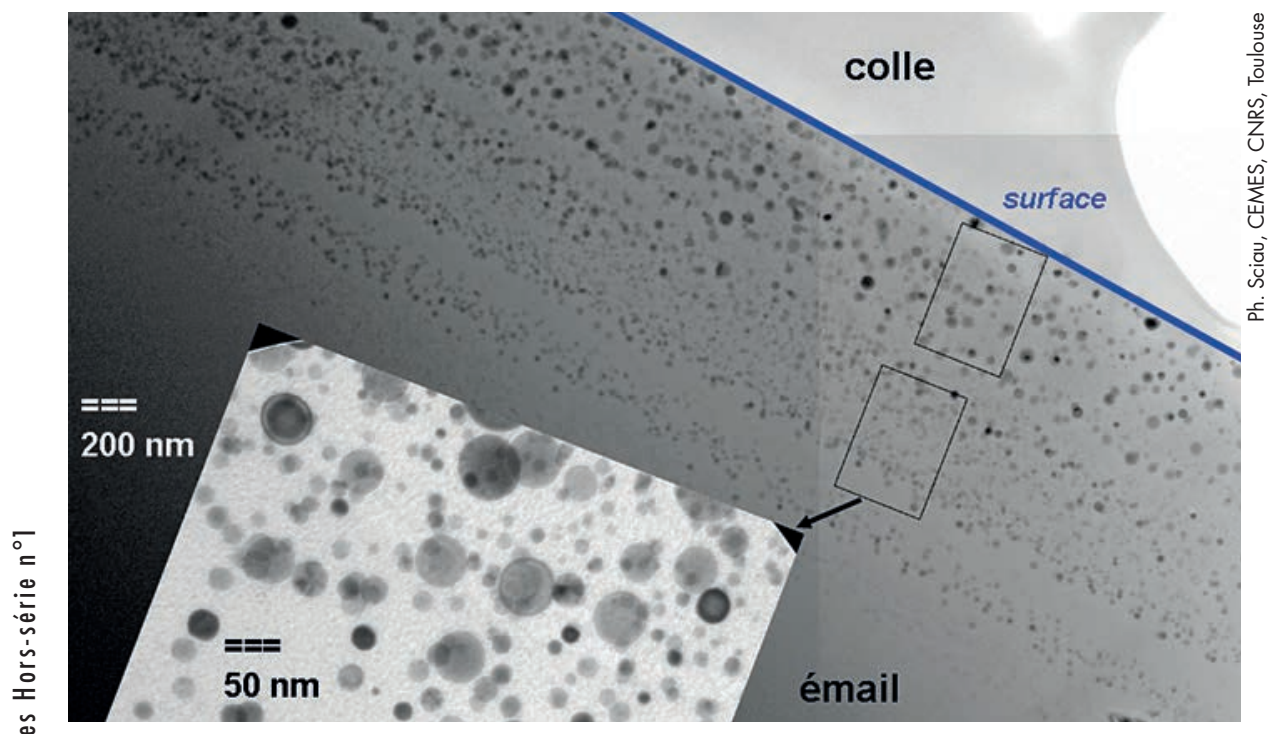

Figure 4. Image en microscopie électronique en transmission d'une coupe de la surface d'une céramique lustrée moderne (potier : Eva Haudum) montrant l'alternance de couches avec ou sans particules de cuivre. Le zoom montre leurs dimensions nanométriques.

fort aujourd'hui par exemple en France chez Alain Dejardin, Eva Haudum ou JeanPaul Van Lith, en Angleterre chez Sutton Taylor, Tony Laverick ou Alan Caiger-Smith et en Espagne Josep Llorens Artigas, Juan Carrillo et Jordi Serra (Colomban, 2004).

\section{Processus de formation des lustres monochromes et polychromes}

Les potiers chimistes du XIX ${ }^{\mathrm{e}}$ siècle, en particulier Théodore Deck, tentèrent de comprendre au moyen d'analyses et par la réalisation de répliques la nature du lustre (Colomban, 2004 ; 2009). Ce n'est que récemment que la structure du lustre, ses mécanismes de synthèse et les différences entre les multiples types ont été compris. La figure 4 montre un cliché en transmission électronique d'une section de céramique lustrée faite par la céramiste Eva Haudum. On observe une alternance de couches de 100 à $400 \mathrm{~nm}$ contenant ou non des nanoparticules métalliques, ici de cuivre, quasi sphériques. La matrice (l'émail) est un silicate amorphe (un verre). D'anciens textes comme celui du potier persan Abû'I-Qâsim' (XIVe siècle) donnent des recettes : sur une céramique déjà émaillée (généralement par un émail au plomb) un mélange d'argile, d'oxydes de fer, de composés organiques (lie de vin... riches en acide acétique) et de sels d'argent et/ou de cuivre est posé là où devra se former le décor lustré. L'objet est ensuite cuit « au rouge » (c'est-à-dire $\sim 600^{\circ} \mathrm{C}$ ) selon l'expression « à deux feux » d'abord en atmosphère réductrice (I'usage de bois vert permet par sa distillation dans l'alandier du four une cuisson au mélange $\mathrm{H}_{2}+\mathrm{CH}_{4}$, très réducteur). L'acide acétique dissout la surface de l'émail, maximisant la surface de réaction. En milieu réducteur les composés organiques se transforment en carbone. Les sels métalliques (nitrates, sulfates) fondent et cela facilite la diffusion des ions argent ou cuivre dans la surface du verre. La présence d'ions à valence multiple et l'alternance de séquences oxydante/réductrice permet l'obtention de couches avec ou sans particules métalliques. On pense que l'exceptionnelle 


\section{Plasmons}

Comme le photon (lumière) et le phonon (vibration atomique) le plasmon est une quasiparticule résultant de la quantification des oscillations collectives d'un gaz d'électrons "libres », comme celvi présent à la surface d'un métal. Dans la plupart des métaux comme des semi-conducteurs, la fréquence des plasmons correspond au domaine de I'ultraviolet, ce qui conduit à une faible réflectivité dans le visible et ainsi à la couleur grise de ces composés. Cependant certains d'entre eux comme l'or et le cuivre, possèdent des transitions inter-bandes électroniques et donc une couleur spécifique. À une interface métal-milieu diélectrique, pour certaines conditions d'illumination l'onde électromagnétique est « absorbée » par les plasmons de surface qui se déplacent à l'interface. La dimension et la forme des particules ainsi que le milieu environnant contrôlent les propriétés optiques du plasmon. L'étude et la mise en œuvre des plasmons de surface, la plasmonique, est un domaine en plein développement tant sur le plan fondamental qu'appliqué ((bio)chimie analytique, dispositifs (opto)électroniques, lithographie, etc.).

polychromie obtenue par certains potiers Abbassides était obtenue empiriquement par le contrôle différencié des températures de surface en réglant la combustion des composés organiques du dépôt contenant les précurseurs. Au refroidissement les résidus du dépôt sont lavés pour révéler le lustre. Les analyses TEM de lustres de différentes périodes et origines (Colomban, 2004 ; Sciau, 2012) démontrent que le nombre de couches, la taille des nanoparticules, leur composition, la présence d'une couche d'impédance sous la surface (couche sans précipités) sont variables et se corrèlent à la qualité et à la couleur du lustre.

\section{Sonder la matière via les particules métalliques: SERS \& VERS}

La présence de nanoparticules métalliques en contact avec un autre milieu a de profondes répercussions sur l'interaction lumière-matière : ainsi lorsqu'un faisceau de lumière monochromatique illumine une nanoparticule d'argent ou d'or « en contact » avec une ou plusieurs molécules, la diffusion inélastique de la lumière (spectrométrie Raman) est exaltée: c'est l'effet SERS (surface-enhanced Raman spectroscopy) méthode largement utilisée pour étudier certains composés en très faible concentration. Cette méthode est efficace si un transfert électronique peut se faire entre la particule et la molécule, par exemple si elle possède des cycles aromatiques. L'étude de verres dichroïques a montré que le même phénomène se produit en volume (volume-enhanced Raman spectroscopy) lorsque les nanoparticules sont dispersées dans un milieu transparent - un verre dans ce cas - pour la longueur d'onde (Colomban \& Schreiber, 2005) : le spectre Raman informe alors seulement sur l'environnement de la particule et non sur l'ensemble de la matrice. Ainsi l'étude des nanomatériaux du passé contribue à une meilleure connaissance de ceux du futur.

\section{Pour en savoir plus}

Ph. Colomban, Secrets retrouvés du Lustre abbasside, La Revue de la Céramique et du Verre, novembre-décembre 2004, 139, 13-21

http://www.glvt-cnrs.fr/ladir/pages/colomban/ lustreceramique.pdf

Ph. Colomban, H. Schreiber, Raman Signature Modification Induced by Copper Nanoparticles in Silicate Glass, J. Raman Spectroscopy 36 (2005) 884-890

$\mathrm{Ph}$. Colomban, The use of metal nanoparticles to produce yellow, red and iridescent colour, from Bronze Age to Present Times in Lustre pottery and glass: Solid state chemistry, spectroscopy and nanostructure, J. Nano Research 8 (2009) 109-132

http://dx.doi.org/10.4028/www.scientific.net/ JNanoR.8.109

Ph. Sciau, Nanoparticles in Ancient Materials: The Metallic Lustre Decorations of Medieval Ceramics, in The Delivery of Nanoparticles, Abbass A. Hashim Ed., INTECHOPEN, 2012

http://www.intechopen.com/books/the-deliveryof-nanoparticles/nanoparticles-in-ancient-materialsthe-metallic-lustre-decorations-of-medieval-ceramics

\section{Symétrie}

\section{METROLOCIE ET POSITIONNEMIENT}

\author{
HEXAPDDE \\ 6 degrés de liberté \\ haute précision \\ configuration du centre de \\ rotation dans le logiciel
}

Hautes performances pour vos positionnements de précision :

optique, instrumentation, spatial, synchrotron...

\section{Nanopos \\ hexapode miniature
résolution $10 \mathrm{~nm}$
courses $\pm 5 \mathrm{~mm}$
charge max. $1 \mathrm{~kg}$ \\ - hauteur $65 \mathrm{~mm}$}

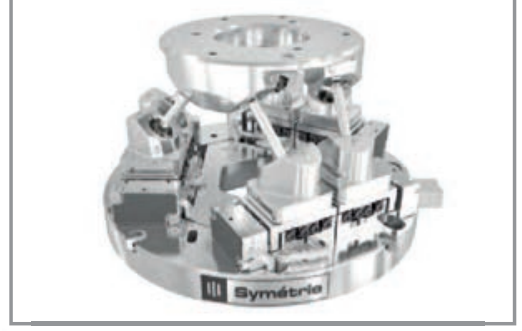

\section{BREVA}

parfait pour des courses et charges plus importantes

résolution $0,5 \mu \mathrm{m}$

courses $\pm 75 \mathrm{~mm}$

charge max. $200 \mathrm{~kg}$

hauteur $350 \mathrm{~mm}$

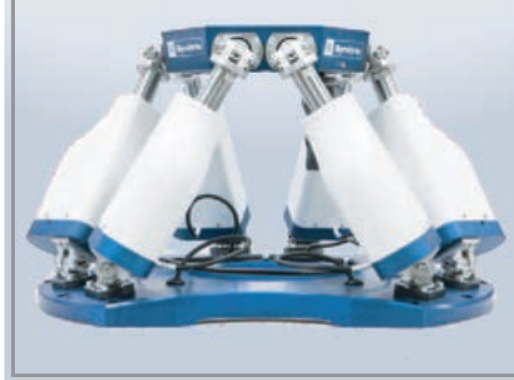

Contact : +33 (0)4 66294388 info@symetrie.fr www.symetrie.fr 